\title{
Changes in pain, swelling, and range of motion according to physical therapy intervention after total knee arthroplasty in elderly patients
}

\author{
Eun-Kyung Noh, Chang-Sik An \\ Department of Physical Therapy, Eulji University, Seongnam, Republic of Korea
}

Objective: This study was carried out to compare changes in pain, swelling and range of motion (ROM) between the two groups according to physical therapy intervention.

Design: Randomized controlled trial.

Methods: There were a total of 20 elderly subjects ages 65-75 years old who have been admitted to the hospital for a total knee arthroplasty. After surgery, the experimental group $(\mathrm{n}=10)$ carried out quadriceps femoris muscle strengthening exercise, hamstring stretching exercises and gastrocnemius stretching exercise with a physical therapist for 30 minutes and additionally, received ice pack therapy for 20 minutes once a day. The control group $(\mathrm{n}=10)$ carried out continuous passive motion for 30 minutes and received ice pack therapy for 20 minutes once a day. The experimental group and control group carried out each intervention program for 2 weeks 3 times a week. The Visual Analogue Scale was used to assess pain, tape measurements were taken to assess swelling, and a steel goniometer was used to assess knee joint ROM.

Results: As a result, the experimental group showed a statistically significant decrease in the pain and swelling, and a significant increase in knee flexion ROM after the intervention, compared to the control group $(p<0.05)$. There was a significant improvement in pain, edema, and knee flexion and extension ROM in all subjects after intervention $(p<0.05)$.

Conclusions: According the results of this study, exercise in the experimental group is effect on the pain, swelling and ROM for total knee arthroplasty.

Key Words: Exercise, Pain, Range of motion, Total knee arthroplasty

\section{Introduction}

Osteoarthritis, the most common form of arthritis, is a degenerative joint disease that causes chronic pain, stiffness, and disability [1], and bone invasion occurs primarily in $80 \%$ of those 55 years or older with a higher prevalence rate in females than in males [2]. Saxon et al. [3] reported that the site of invasion in arthritis can involve any joint; however, the knee joint has the highest rate of occurrence [4]

A normal knee joint consists of smooth cartilage, allowing the cartilage to move with almost no friction. However, a damaged joint facet is rough and irregular, which can cause excruciating pain. Moreover, exacerbation of arthritis can lead to joint deformation and stiffness. A total knee arthroplasty (TKA) is performed to address these problems [5].

Han [6] reported that TKA, as a procedure that replaces the damaged joint facet with an artificial structure similar to the knee joint, After a TKA, the functional status of the body is severely degraded due to the reduced strength and joint range of motion (ROM) and extreme pain caused by the surgery. It calls for exercise programs for enhancing the functional status, especially for seniors. They are likely to have many severe complications after a surgery [7].

Naso et al. [8] indicated that after TKA, muscle strength

Received: 24 November, 2015 Revised: 2 December, 2015 Accepted: 7 December, 2015

Corresponding author: Chang-Sik An

Department of Physical Therapy, Eulji University, 553 Sanseong-daero, Sujeong-gu, Seongnam 13135, Republic of Korea

Tel: 82-31-740-7232 Fax: 82-31-740-7367 E-mail: acsik@eulji.ac.kr

(c) This is an Open-Access article distributed under the terms of the Creative Commons Attribution Non-Commercial License (http://creativecommons.org/licens es/by-nc/4.0) which permits unrestricted non-commercial use, distribution, and reproduction in any medium, provided the original work is properly cited.

Copyright $@ 2015$ Korean Academy of Physical Therapy Rehabilitation Science 
can be reduced from the incision of the quadriceps, which plays an important role in lower-extremity function, and even more severe pain can develop. On the other hand, Stevens et al. [9] reported that if the quadriceps fails to recover within the first month after surgery, the patient will experience persistent weakening of the muscles. Therefore, early implementation of muscle strengthening exercises for the quadriceps is one of the important factors that determine the success of TKA [10].

Recovery of muscle strength in the quadriceps is very important for weight bearing, stability, and normal functioning of the knee joints [11,12]. Quadriceps setting exercise (QSE) is one of the primary muscle strengthening exercises designed for early muscle strengthening, through the isometric contraction of the quadriceps in patients who have postoperative knee joint dysfunction. In a study that compared QSE with electrical stimulation, it was reported that QSE was more effective in increasing muscle strength [13].

According to studies on continuous passive motion (CPM), CPM extends the ROM in the joints in the early stages after surgery [14]. However, CPM has the disadvantages of requiring a lengthy treatment period and high costs associated with equipment requirements. Furthermore, there have been reports on CPM causing postoperative pain and discomfort in some patients [15].

Edema that develops at the surgical site after TKA often occurs during early stage of the surgery. In severe cases of edema, blisters can form and damage the skin; early ambulation can be hindered, thus delaying recovery; and pain from edema may occur when starting rehabilitation [16]. Cold therapy is known to induce the contraction of peripheral blood vessels, which, in turn, reduces the temperature in the damaged area. Through this mechanism, edema is controlled through the lowering of cellular permeability, hemorrhaging, and tissue metabolism; in addition, it has the effect of changing the pain transmission from the damage muscle and reducing muscle contraction as a response to such change [17].

Cold therapy is widely used in clinical settings owing to its cost-effectiveness, relative safety, and ease of use compared with other interventions. However, the application of cold therapy on TKA patients has been limited to only a few cases. As there is a lack of studies on the effects of cold therapy on edema, such studies are needed in TKA patients by using cold therapy that has been proven to be effective in alleviating edema. Based on the theories above, this study intends to solve the medical issues of the patients who undergo
TKA more effectively. First, ice packs were applied in order to reduce their swelling. As for the experimental group, they engaged in quadriceps femoris muscle strengthening and hamstring and gastrocnemius stretching exercises in a systematic manner. There is a need to verify the effects in a short period of time in comparison with the CPM applied to the control group.

Therefore, the objective of the present study was to investigate the changes in pain, edema, and ROM in the knee joints of elderly patients according to the post-TKA physical therapy intervention modality, and to use the findings to provide basic data for deciding the intervention modalities and application period for TKA patients.

\section{Methods}

\section{Study subjects}

The present study was conducted after receiving the consent form for study participation from the subject or the subject's guardian after sufficient explanation on the study content and objectives was given. The subjects consisted of 20 elderly patients, $65-75$ years old, who had concomitant pain and edema, and were hospitalized to undergo TKA at Eulji General Hospital in Nowon district of Seoul, South Korea, between May 4 and June 26, 2015. The criteria for selecting the subjects are as follows. First, those who have received a TKA within the last week. Second, those who have more than a moderate level of pain (visual analogue scale [VAS] 5) on the lower limbs. Third, those who have no medical conditions other than an osteoarthritis on the lower limbs.

\section{Study design}

The present study was conducted on patients with a diagnosis of degenerative knee osteoarthritis and hospitalized to undergo TKA, with 10 patients each assigned to the experimental group and the control group. On day 1 , the control group performed 30 minutes of CPM, followed by 20 minutes of ice packing, whereas the experimental group performed 30 minutes of hamstring stretching, gastrocnemius muscle stretching, and quadriceps femoris muscle strengthening exercises with a physical therapist, followed by $20 \mathrm{mi}-$ nutes of ice packing. The intervention programs for the experimental and control groups consisted of three sessions per week for 2 weeks. A total of three measurements were taken before the intervention and at days 7 and 14 of after the intervention. 
Physical therapy intervention method for the control group

\section{CPM}

CPM (CPS-1000 Stratek; State Korea, StraTek, Anyang, Korea) was performed with the subject in a supine position, and the passive motion consisted of a single cycle of flexion and extension with a speed of 30 seconds per cycle with the movement maintained at that speed. The CPM was set at a motion within a range that did not cause pain to the patient [18].

\section{Ice pack}

An ice pack was applied directly to the affected area for 20 minutes with the patient in a supine position (Figure 1).

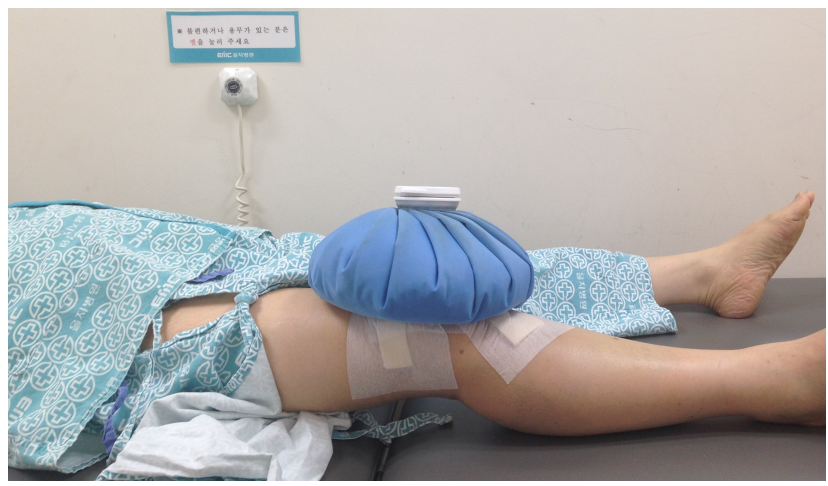

Figure 1. Ice pack.

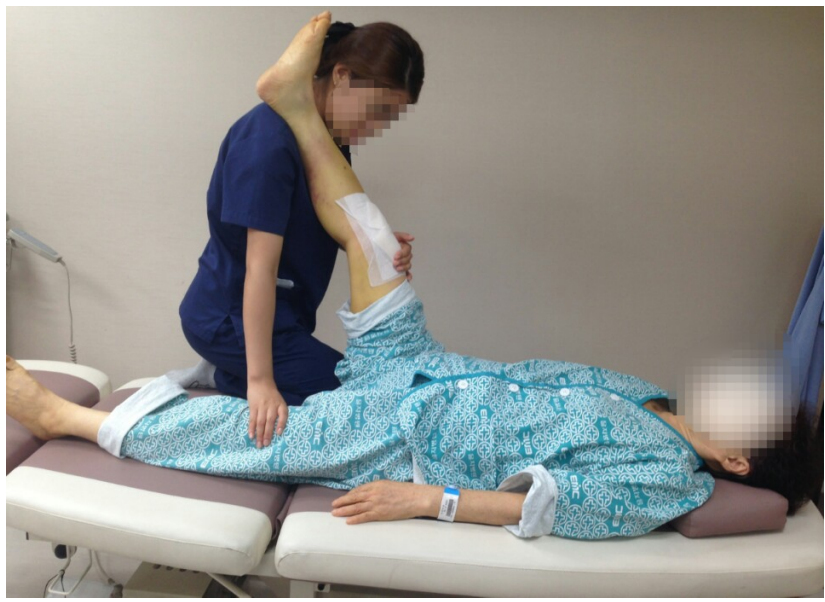

Figure 2. Hamstring stretching exercise.
Physical therapy intervention method for the experimental group

\section{Hamstring stretching exercise}

Straight leg raising was performed by the subject by raising the operation leg from the floor while in a supine position. The therapist's hand held the non-operated leg to prevent it from being lifted (Figure 2) [18].

Quadriceps femoris muscle strengthening exercise

(1) The subject, while in a supine position, lifted the heel

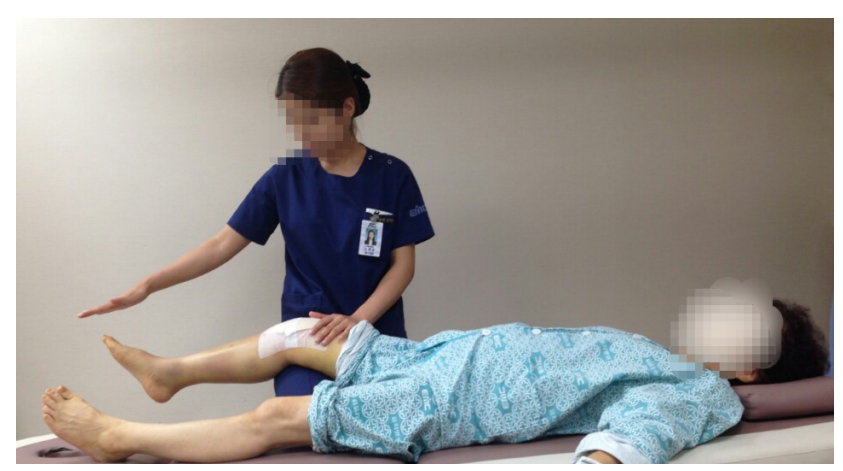

Figure 3. Quadriceps femoris muscle strengthening exercise-1.

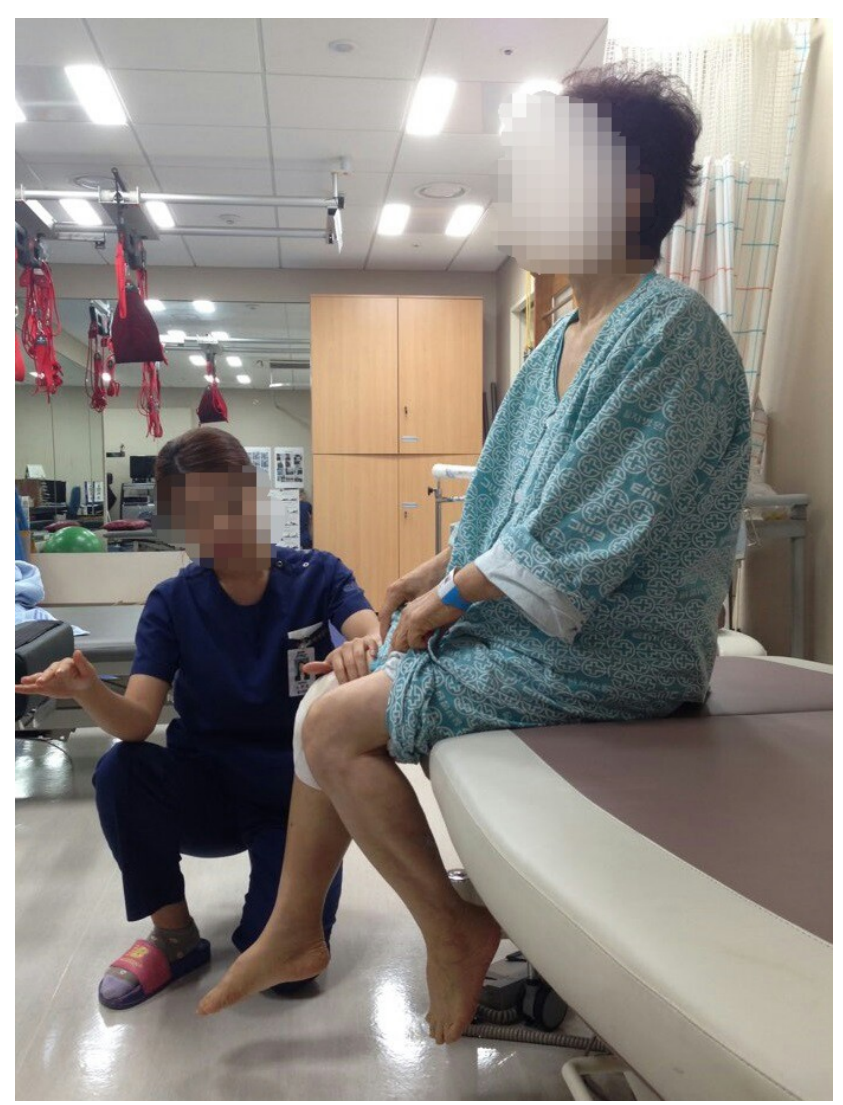

Figure 4. Quadriceps femoris muscle strengthening exercise-2. 
from the floor, with the area below the knee being kept in extension the therapist's hand or with a towel. After keeping the knee fully extended for 10 seconds, the heel was slowly placed back down on the floor (Figure 3 ).

(2) While in an upright sitting position, the subject lifted the operation leg to reach the therapist's hand at a set height, which was given as a gravity resistance exercise given as a task for knee joint flexion (Figure 4).

\section{Gastrocnemius muscle stretching exercise}

In a standing position, the subject placed the front portion of the sole on top of a wedge and performed the exercise while focusing on flexing the hip and knee joints (Figure 5) [18].

\section{Ice pack}

Ice pack was applied directly to the affected area for 20 minutes with the patient in a supine position (Figure 1).

\section{Measurement methods}

After applying the physiotherapy intervention to the control group and experimental group, their pain, swelling, and joint ROM were measured as follows.

\section{Pain measurement}

Pain was measured by using a $10 \mathrm{~cm}$ horizontal VAS, with

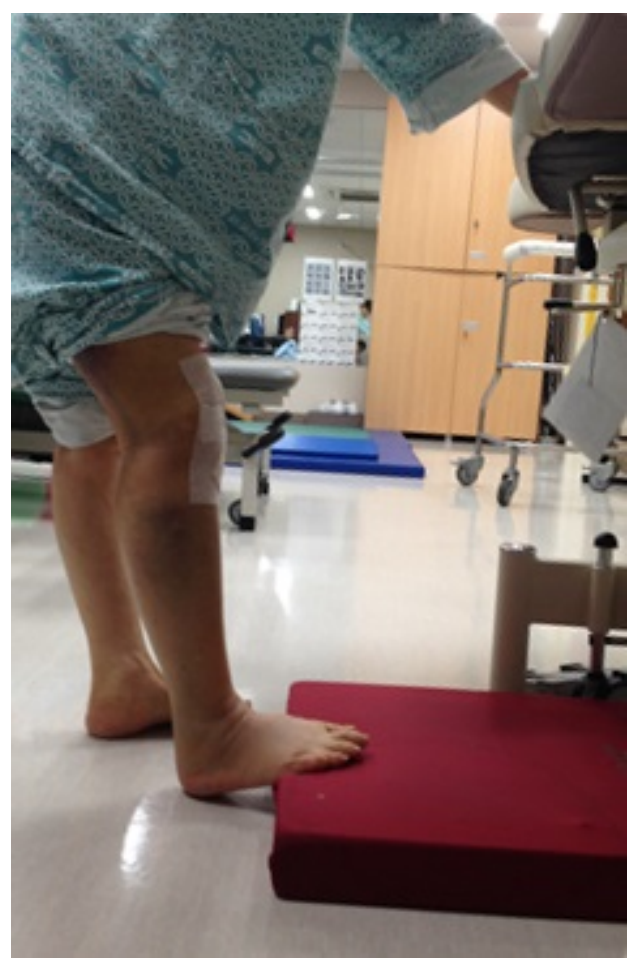

Figure 5. Gastrocnemius muscle stretching exercise. the left end representing 'no pain' ( 0 point $)$ and the right end representing 'very severe pain' (10 points). The subject marked the pain felt on the scale with an arrow. A higher score indicated a greater pain severity. The data analysis revealed that the intertester reliability $(\mathrm{r}=0.62$; intraclass correlation coefficient $[\mathrm{ICC}]=0.75)$ were high [19].

\section{Edema measurement}

In the present study, edema was quantified according to the changes in the circumference of the lower extremity. For this evaluation, a tape measurement method that is commonly used to evaluate an increase in lower extremity volume was used. To reduce measurement errors, the mean value from three repeated measurements was used.

The measurement method involved using a double-sided tape measure with width of $10 \mathrm{~mm}$ and a length of $150 \mathrm{~m} \mathrm{(60}$ in) to measure the circumference of the area $5 \mathrm{~cm}$ above the knee joint line, while the subject was in a supine position [20].

\section{Joint angle measurement}

The ROM of the knee joint of the subject was measured by using a steel goniometer (Steel Goniometer Stainless, BaseLine; Patterson Medical, Warrenville, IL, USA). To reduce measurement errors, the mean value from three repeated measurements was used.

To measure the flexion and extension angles of the knee joint, the subject was placed in a supine position, and the maximum degree of flexion and extension was measured with the fixed arm placed in between the greater trochanter of the femur and the middle portion of the femur, and the moving arm positioned in a straight line from the fibular head to the lateral malleolus of the ankle. The data analysis revealed that the intertester reliability $(r=0.98 ; \mathrm{ICC}=0.99)$ were high [21].

\section{Data analysis}

Statistical analyses of the measured data in the present study were performed by using the Windows version of PASW ver.18.0 (IBM Co., Armonk, NY, USA). An independent t-test was used for the intergroup comparison of the differences in knee joint pain, edema, and ROM. For the intergroup comparison of the pattern of change in knee joint pain, edema, and ROM, a one-way repeated ANOVA that has a between-subjects factor was used. The statistical significance level was set at $\alpha=0.05$. 


\section{Results}

\section{General characteristics of the subjects}

Among the subjects, there were 7 males and 13 females, with a mean age of 68.6 years. The mean height and weight was $164.1 \mathrm{~cm}$ and $61.1 \mathrm{~kg}$, respectively, and six subjects had received a TKA on the left side, whereas 14 had received a TKA on the right side. The general characteristics of the experimental and control groups are given in Table 1 .

\section{Intergroup comparison on level of pain}

Analyses were performed to examine how the differences in pain levels changed according to the measurement period (Table 2).

Although there were no significant intergroup differences at pre-intervention and at day 7 of the intervention, a sig- nificant intergroup difference was found on day 14 of the intervention $(p<0.001)$. Therefore, it can be stated that pain according to the time was different between the two groups $(p<0.001)$.

\section{Intergroup comparison on the level of edema}

Analyses were performed to examine how the differences in pain changed according to the measurement period (Table 2).

Although there were no significant intergroup differences at pre-intervention and on day 7 of the intervention, a significant intergroup difference was found on day 14 of the intervention $(p<0.05)$. Therefore, it can be stated that the level of edema was different between the two groups according to the time period $(p<0.001)$.

Table 1. General and clinical characteristics of the participants

$(\mathrm{N}=20)$

\begin{tabular}{|c|c|c|c|c|}
\hline Variable & $\begin{array}{l}\text { Experimental group } \\
\qquad(\mathrm{n}=10)\end{array}$ & $\begin{array}{l}\text { Control group } \\
(\mathrm{n}=10)\end{array}$ & $\mathrm{t}$ & $p$ \\
\hline Sex (male/female) & $3 / 7$ & $4 / 6$ & 0.220 & 0.639 \\
\hline Age (yr) & $68.70(2.87)$ & $68.50(2.64)$ & 0.162 & 0.873 \\
\hline Height $(\mathrm{cm})$ & $175.00(7.20)$ & $163.70(8.00)$ & 0.235 & 0.817 \\
\hline Weight $(\mathrm{kg})$ & $59.20(8.98)$ & $62.90(8.05)$ & -0.970 & -0.345 \\
\hline Operated side (left/right) & $3 / 7$ & $3 / 7$ & 0.000 & 1.000 \\
\hline Pain (point) & $6.70(1.16)$ & $7.00(0.82)$ & -0.669 & 0.512 \\
\hline Swelling (cm) & $40.10(3.60)$ & $40.40(5.02)$ & -0.154 & 0.880 \\
\hline Flexion ROM $\left(^{\circ}\right)$ & $59.00(6.99)$ & $52.50(7.17)$ & 2.053 & 0.055 \\
\hline Extension ROM $\left(^{\circ}\right)$ & $-4.5(4.4)$ & $-3.5(4.1)$ & -0.526 & 0.605 \\
\hline
\end{tabular}

Values are presented as number only or mean (SD).

ROM: range of motion.

Table 2. Compared pain, swelling and range of motion (ROM) between groups

\begin{tabular}{|c|c|c|c|c|}
\hline & Vaiable & Pre-intervention & Day 7 & Day 14 \\
\hline \multirow[t]{3}{*}{ Pain (point) } & Experimental group $(n=10)$ & $6.70(1.16)$ & $5.50(0.71)$ & $2.80(0.79)$ \\
\hline & Control group $(n=10)$ & $7.00(0.82)$ & $6.00(0.94)$ & $4.20(0.79)$ \\
\hline & $\mathrm{t}$ & -0.669 & -1.342 & $-3.969^{* * *}$ \\
\hline \multirow[t]{3}{*}{ Swelling (cm) } & Experimental group $(n=10)$ & $40.10(3.60)$ & $36.30(3.74)$ & $31.40(3.27)$ \\
\hline & Control group $(n=10)$ & $40.40(5.02)$ & $37.80(4.42)$ & $35.90(4.25)$ \\
\hline & $\mathrm{t}$ & -0.154 & -0.819 & $-2.651^{*}$ \\
\hline \multirow[t]{3}{*}{ Flexion $\operatorname{ROM}\left(^{\circ}\right)$} & Experimental group $(n=10)$ & $59.00(6.99)$ & $98.50(6.69)$ & $118.50(2.42)$ \\
\hline & Control group $(n=10)$ & $52.50(7.17)$ & $89.00(6.58)$ & $110.00(5.27)$ \\
\hline & $\mathrm{t}$ & 2.053 & $3.201^{* *}$ & $0.927^{* * *}$ \\
\hline \multirow[t]{3}{*}{ Extension $\mathrm{ROM}\left({ }^{\circ}\right)$} & Experimental group $(n=10)$ & $-4.5(4.4)$ & $-3.0(3.5)$ & $0(0)$ \\
\hline & Control group $(n=10)$ & $-3.5(4.1)$ & $-2.5(2.6)$ & $0(0)$ \\
\hline & $\mathrm{t}$ & -0.526 & -0.361 & $0(0)$ \\
\hline
\end{tabular}

Values are presented as mean (SD).

${ }^{*} p<0.05, \stackrel{* *}{p}<0.01,{ }^{* * *} p<0.001$. 


\section{Intergroup comparison on the degree of flexion ROM}

Analyses were performed to examine how the differences in flexion ROM changed according to the measurement period (Table 2).

Although there were no significant intergroup difference at pre-intervention, significant intergroup differences were found on day 7 and day 14 of the intervention $(p<0.01$, $p<0.001$ ). Therefore, it can be stated that the flexion ROM was different between the two groups according to period $(p<0.001)$.

\section{Intergroup comparison on the degree of extension ROM}

Analyses were performed to examine how the differences in extension ROM changed according to the measurement period (Table 2).

There were no significant intergroup differences at preintervention and on day 7 and day 14 of the intervention $(p<0.001)$.

\section{Clinical variables compared time progressive of the ex- perimental group}

The pain scores decreased significantly after surgery $(p<0.05)$. The post hoc test results using the Fisher's least significant difference reveals that pain during the pre-surgical state was significantly greater than on day 7 and day 14 $(p<0.05)$. There was a significant decrease in edema after surgery $(p<0.05)$. Edema during the pre-surgical state was significantly greater than on day 7 and day $14(p<0.05)$. Knee flexion and extension ROM was significantly increased after surgery. Knee flexion and extension ROM was significantly greater 14 days following surgery than during the pre-surgical state and on day $7(p<0.05)$ (Table 3$)$.

\section{Clinical variables compared time progressive of the con- trol group}

Pain scores were decreased significantly after surgery $(p<0.05)$. Edema was also decreased significantly after surgery $(p<0.05)$. Pain and swelling during the pre-surgical state was significantly greater than on day 7 and day 14 $(p<0.05)$. Knee flexion and extension ROM was significantly increased after surgery $(p<0.05)$. Knee flexion and extension ROM was significantly greater on day 14 following surgery compared to the pre-surgical state and on day $7(p<0.05)$ (Table 3).

\section{Discussion}

The present study examined the effects of two different physical therapy intervention on knee joint pain, edema, and ROM by dividing 20 patients who had received TKA into the experimental and control groups. Patients who have had osteoarthritis for a long time can develop gait disorders from severe pain and degenerative deformation [22], and regardless of how successful the TKA may be, gait disturbance can occur owing to the insufficient support in the joint because of atrophy or weakening of the surrounding muscles [23]. Therefore, it is necessary to prevent muscle atrophy and increase muscle strength through systematic stretching and muscle strengthening exercises immediately after surgery. Accordingly, the results showed that the stretching and muscle strengthening exercises used in the present study were effective for the patients.

In the present study, the examination of the effects on pain according to time between the two groups showed a significant pain reduction in both groups over time. Although there were no significant intergroup differences at pre-intervention and on day 7 of the intervention, whereas a significant intergroup difference was observed on day 14 of the intervention. These findings were similar to the results from precedent studies by Shin [24], in which a rehabilitation program for 5 weeks for patients who underwent TKA resulted in a significant reduction in pain in the experimental group compared with that in the control group, and that by

Table 3. Pain, swelling, and range of motion in flexion and extension of the participants

$(\mathrm{N}=20)$

\begin{tabular}{|c|c|c|c|c|c|c|c|c|}
\hline \multirow{2}{*}{ Variable } & \multicolumn{3}{|c|}{ Experimental group $(n=10)$} & \multirow{2}{*}{$p$} & \multicolumn{3}{|c|}{ Control group $(n=10)$} & \multirow{2}{*}{$p$} \\
\hline & Pre-intervention & Day 7 & Day 14 & & Pre-intervention & Day 7 & Day 14 & \\
\hline Pain (point) & $6.70(1.16)$ & $5.50(0.71)$ & $2.80(0.79)$ & $<0.001$ & $7.00(0.82)$ & $6.00(0.94)$ & $4.20(0.79)$ & $<0.001$ \\
\hline Swelling $(\mathrm{cm})$ & $40.10(3.60)$ & $36.30(3.74)$ & $31.40(3.27)$ & $<0.001$ & $40.40(5.02)$ & $37.80(4.42)$ & $35.90(4.25)$ & $<0.001$ \\
\hline Flexion $\left({ }^{\circ}\right)$ & $59.00(6.99)$ & $98.50(6.69)$ & $118.50(2.42)$ & $<0.001$ & $52.50(7.17)$ & $89.00(6.58)$ & $110.00(5.27)$ & $<0.001$ \\
\hline Extension $\left(^{\circ}\right)$ & $-4.50(4.40)$ & $-3.00(3.50)$ & $0.00(0.00)$ & 0.045 & $-3.50(4.10)$ & $-2.50(2.60)$ & $0.00(0.00)$ & 0.020 \\
\hline
\end{tabular}

Values are presented as mean (SD). 
Bruun-Olsen et al. [15] in which providing CPM after TKA had no positive effect when compared with applying only active movement for pain, ROM, and gait ability at the first week and 3 months after surgery. Moreover, Han [6] applied a ground rehabilitation program with three sessions per week for 6 weeks, and reported a significant reduction in pain, similar to the results from previous studies. Therefore, it was determined that the exercise program applied to the experimental group in the present study had a positive effect on pain reduction.

In general, edema develops early in surgery, and consequently, patients complain of pain and discomfort from edema when starting an early rehabilitation program $[16,25]$. Accordingly, cold therapy is considered an effective treatment method resulting in a speedy recovery and the alleviation of edema and pain in the patients. Therefore, in the present study, we applied cold therapy on both groups for the reduction of edema and pain. As a result, both groups showed a significant reduction in edema over time, measured on the basis of the leg circumference at $5 \mathrm{~cm}$ above the knee. However, there was no significant intergroup difference at pre-intervention and on day 7 of the intervention, whereas a significant intergroup difference was observed on day 14 of the intervention. The findings in the present study were consistent with the results from the study by Chesterton et al. [26]. in which the exercise program and cold therapy applied to the experimental group were shown to be effective in reducing edema and pain.

In the present study, examination of the effects on ROM between the two groups according to time showed a significant increase in ROM in both groups as time elapsed. Although there was no significant intergroup difference at pre-intervention, significant intergroup differences were found on day 7 and day 14 of the intervention. These findings were consistent with precedent studies that reported that CPM, as compared to active exercise, had no effect on the ROM at 1 week after surgery [14,27,28]. These results therefore show that an exercise program applied to the experimental groups was more effective than CPM in increasing the flexion angle within a short period time. Although both groups showed the extension ROM reaching the normal ROM level at day 14 of the intervention, there was no significant intergroup difference compared to that at pre-intervention.

As shown above, improvements in pain, edema, and ROM over time were seen in both groups of elderly patients according to the intervention method used after TKA. In comparison to pre-intervention, significant differences in pain, edema, and flexion ROM were seen in the experimental group compared with those in the control group, at 2 weeks after intervention. Moreover, improvements in the extension ROM were seen in both groups at 2 weeks after the intervention, as compared to that at pre-intervention; however, the difference was not significant.

The limitations of this study are as follows. First, there was a small number of subjects. Second, the exercise period was not long enough, because most patients are released from a university hospital within 3 weeks. Third, the experiment was conducted in one hospital only and thus it is problematic to expand the findings for broader interpretation.

The present study was a quasi-experimental research that used a one-way repeat ANOVA to test the mean differences between the experimental and control groups with regard to measurements of pain, edema, and ROM, taken at pre-intervention and on day 7 and day 14 of the intervention after applying an exercise program on elderly patients who were hospitalized to receive TKA for degenerative knee osteoarthritis, with the program starting from postoperative day 4 and lasting for 2 weeks.

The results showed that in the comparisons between the experimental and control groups, reduction in pain and edema was seen according to the elapsed time, whereas flexion ROM showed a significant increase. Moreover, improvements in the extension ROM were seen in both groups over time, as compared with that immediately after surgery; however, the improvement in the experimental group was greater than that in the control group. Nevertheless, the difference between the two groups was not significant.

On the basis of the above results, it was determined that implementation of an exercise program immediately after surgery was effective for improving physical functions in TKA patients. Therefore, systematically applying stretching and muscle strengthening exercises on TKA patients immediately after surgery can assist to a more timely return to independent daily living.

\section{Conflict of Interest}

The authors declared no potential conflicts of interest with respect to the authorship and/or publication of this article. 


\section{References}

1. Hinton R, Moody RL, Davis AW, Thomas SF. Osteoarthritis: diagnosis and therapeutic considerations. Am Fam Physician 2002;65:841-8.

2. Farquhar SJ, Reisman DS, Snyder-Mackler L. Persistence of altered movement patterns during a sit-to-stand task 1 year following unilateral total knee arthroplasty. Phys Ther 2008;88:567-79.

3. Saxon L, Finch C, Bass S. Sports participation, sports injuries and osteoarthritis: implications for prevention. Sports Med 1999; 28:123-35.

4. Brooks PM. Impact of osteoarthritis on individuals and society: how much disability? Social consequences and health economic implications. Curr Opin Rheumatol 2002;14:573-7.

5. Noble PC, Gordon MJ, Weiss JM, Reddix RN, Conditt MA, Mathis KB. Does total knee replacement restore normal knee function? Clin Orthop Relat Res 2005;(431):157-65.

6. Han J. Effectiveness of 8 weeks of application of aqua complexed exercise on lower limbs muscular strength, pain relief and balance after total knee arthroplasty [Master's degress]. Seoul: Korea University; 2010.

7. Kramer JF, Speechley M, Bourne R, Rorabeck C, Vaz M. Comparison of clinic- and home-based rehabilitation programs after total knee arthroplasty. Clin Orthop Relat Res 2003;(410):22534.

8. Naso F, Carner E, Blankfort-Doyle W, Coughey K. Endurance training in the elderly nursing home patient. Arch Phys Med Rehabil 1990;71:241-3.

9. Stevens JE, Mizner RL, Snyder-Mackler L. Quadriceps strength and volitional activation before and after total knee arthroplasty for osteoarthritis. J Orthop Res 2003;21:775-9.

10. Greene KA, Schurman JR 2nd. Quadriceps muscle function in primary total knee arthroplasty. J Arthroplasty 2008;23(7 Suppl):15-9.

11. MacDonald SJ, Bourne RB, Rorabeck CH, McCalden RW, Kramer J, Vaz M. Prospective randomized clinical trial of continuous passive motion after total knee arthroplasty. Clin Orthop Relat Res 2000;(380):30-5.

12. Yim SJ, Min KD, Lee YK, Kim HT, Kang HK. Efficacy of physiotherapist after total knee arthroplasty. J Korean Knee Soc 2009;21:258-64.

13. Choi JC, Han DU. The effects of quadriceps setting exercise and electrical stimulation on improvement of quadriceps muscle strength. J Korean Soc Phys Ther 2001;13:273-80.

14. Beaupré LA, Davies DM, Jones CA, Cinats JG. Exercise combined with continuous passive motion or slider board therapy compared with exercise only: a randomized controlled trial of patients following total knee arthroplasty. Phys Ther 2001;81: 1029-37.

15. Bruun-Olsen V, Heiberg KE, Mengshoel AM. Continuous passive motion as an adjunct to active exercises in early rehabilitation following total knee arthroplasty: a randomized controlled trial. Disabil Rehabil 2009;31:277-83.

16. Barry S, Wallace L, Lamb S. Cryotherapy after total knee replacement: a survey of current practice. Physiother Res Int 2003; 8:111-20.

17. Mora S, Zalavras CG, Wang L, Thordarson DB. The role of pulsatile cold compression in edema resolution following ankle fractures: a randomized clinical trial. Foot Ankle Int 2002;23: 999-1002.

18. Lee SH. The effects of physical therapy intervention methods on range of motion, muscular strength, and balance after total knee replascement [Master's degress]. Kwangju: Kwangju Woman's University; 2014.

19. Huskisson EC. Measurement of pain. J Rheumatol 1982;9:768-9.

20. Shim EO. A study on the effects of structured cryotherapy on pain, Inflammation, range of motion and edema in patients with total knee arthroplasty [Master's degress]. Kwangju: Chonnam National University; 2014.

21. Gogia PP, Braatz JH, Rose SJ, Norton BJ. Reliability and validity of goniometric measurements at the knee. Phys Ther 1987; 67:192-5.

22. Park HO, Park KS. A study on experiences of total knee replacement in patients with osteoarthritis. J Muscle Joint Health 1996; 3:135-50.

23. Yoo MC. New perspectives of treatment of osteoarthritis. J Muscle Joint Health 1995;2:227-9.

24. Shin JY. The effect of rehabilitation program on pain, ROM of the knee joint, and mobility in elderly with total knee arthroplasty [Master's degress]. Busan: Dongeui University; 2011.

25. Healy WL, Seidman J, Pfeifer BA, Brown DG. Cold compressive dressing after total knee arthroplasty. Clin Orthop Relat Res 1994;(299):143-6.

26. Chesterton LS, Foster NE, Ross L. Skin temperature response to cryotherapy. Arch Phys Med Rehabil 2002;83:543-9.

27. Chiarello CM, Gundersen L, O'Halloran T. The effect of continuous passive motion duration and increment on range of motion in total knee arthroplasty patients. J Orthop Sports Phys Ther 1997;25:119-27.

28. Kumar PJ, McPherson EJ, Dorr LD, Wan Z, Baldwin K. Rehabilitation after total knee arthroplasty: a comparison of 2 rehabilitation techniques. Clin Orthop Relat Res 1996;(331):93101. 